\title{
Use of Portable Global Positioning System (GPS) Devices in Exposure Analysis for Time-location Measurement
}

\author{
Kiyoung Lee ${ }^{\dagger} \cdot$ Joung Yoon Kim $\cdot$ Kiran Putti* ${ }^{*}$ Deborah H. Bennett** • \\ Diana Cassady** - Irva Hertz-Picciotto** \\ Graduate School of Public Health and Institute of Health and Environment, Seoul National University, \\ 28 Yungeun-dong, Jongro-gu, Seoul 110-460, Korea \\ *College of Public Health, University of Kentucky, 121 Washington Avenue, Lexington, Kentucky, 40536, USA \\ **Department of Public Health Sciences, University of California, Davis, One Shields Ave, \\ Davis, California, 95616, USA
}

(Received October 11, 2009/Revised November 12, 2009/Accepted November 29, 2009)

\begin{abstract}
Exposure analysis is a critical component of determining the health impact of pollutants. Global positioning systems (GPS) could be useful in developing time-location information for use in exposure analysis. This study compares four low cost GPS receivers with data logging capability (Garmin 60, Garmin Forerunner 201, GeoStats GeoLogger and Skytrx minitracker MT4100) in terms of accuracy, precision, and ease of use. The accuracy of the devices was determined at two known National Geodetic Survey points. The coordinates logged by the devices were compared when the devices were carried while walking and driving. The Garmin 60 showed better accuracy and precision than the GeoLogger when they were placed at the geodetic points. The Forerunner and Skytrx did not record when they were kept stationary. When the subject wore the devices while walking, the location of the devices differed by about $8 \mathrm{~m}$ on average between any two device combinations involving the four devices. The distance between the coordinates logged by the devices decreased when the devices were carried with their antennas facing the sky. All the devices showed similar routes when they were used in a car. All the devices except the Forerunner had satisfactory signal reception when they were worn and when they were carried in the car. The GeoLogger is less comfortable for the subject because of specific wearing requirements. This evaluation found that the Garmin 60 and the Skytrx may be useful in personal exposure analysis studies to record time-location data.
\end{abstract}

Keywords: personal exposure, time-activity pattern, GPS, accuracy, performance

\section{Introduction}

Exposure assessment is a critical component of determining the health impact of pollutants and has been a subject of investigation for environmental scientists. Exposure is defined as contact over time and space between a person and one or more biological, chemical, or physical agents. Exposure analysis identifies and defines the exposures that occur, or are anticipated to occur, in human

Corresponding author: Graduate School of Public Health and Institute of Health and Environment, Seoul National University

Tel: 82-2-740-8881, Fax: 82-2-745-9104

E-mail: cleanair@snu.ac.kr populations. Because exposure is directly proportional to the duration of exposure, the accuracy in recording the time-location influences the accuracy of the exposure calculation. Relative to concentration measurement, development of new methods for measuring location has been slower.

Methods used for acquiring time-location information include time-activity diaries, questionnaires, and observation. ${ }^{1}$ These conventional methods can theoretically give the subject's location both indoors and outdoors. However, these methods rely heavily on recall abilities and the voluntary participation of subject. The quality of the data can be questionable due to the differences among individuals' motivation to participate and their 
recall of events. It is highly impractical to ask subjects to record their exact outdoor locations over a period of time. Some researchers have suggested direct observation of subjects to record their time-location. ${ }^{2)}$ This can be done in studies with a small number of subjects over a limited period of time, but only at the expense of the subjects' privacy.

Global positioning system (GPS) is a new technology with the potential to address shortcomings inherent in conventional measures of timelocation. GPS has been available to the general public for navigation since 2000. GPS determines the position of the receiver by receiving radio signals from 24 satellites and 3 back-up satellites which revolve around the earth twice a day in predetermined orbits. The 24 satellites revolve in 6 orbits with 4 satellites in each orbit. These satellites are operated by the Navigational System with Timing and Ranging (NAVSTAR), are maintained by the US Department of Defense, and are available for use throughout the world. ${ }^{3)}$ To determine the location of the receiver on the ground, the receiver needs to "see" at least 3 satellites to get a two-dimensional location and 4 or more for a three-dimensional location. ${ }^{4)}$ The GPS technology provides the convenience of recording time location data with minimal human intervention, thus eliminating error from human sources. The GPS receivers can log the location of the subject in the form of coordinates (latitude and longitude). The location can be determined by mapping the coordinates in any Geographic Information System (GIS) software.

Several studies have used portable GPS devices in exposure assessment. ${ }^{5-7)}$ GPS was used to track adult subjects' location, and the logged data was compared to the time-activity diaries of the subjects. ${ }^{7)}$ There was high agreement between GPS location and diaries, with at least one additional location identified by GPS compared to the diary. Although location was measured accurately within 10 $\mathrm{m}$, the GPS units recorded only $30 \%$ of the monitoring time attempted primarily due to short battery life. In a study involving the time-activity of children, the resolution, feasibility, interference, and reception of two GPS-personal acquisition logger (GPS-PAL) receivers were evaluated. ${ }^{5)}$ The
GPS receivers had wiring problems and issues with the continuously operating reference stations (CORS) base-file correction in post processing the data. In addition, the receiver they used was a three-piece set-up implanted into a vest, which might not be comfortable to wear in all weather conditions. In another study, data from a differentially corrected GPS (dGPS) tracking device worn by children ages 3-5 years old was compared to the National Human Exposure Assessment Survey (NHEXAS) diary timeline completed by parents. ${ }^{\text {.) }}$ The study demonstrated that automated tracking by GPS can improve the quality and collection efficiency of time-location data. Significant advances have been made in the GPS devices since the studies were done.

Use of the GPS in exposure assessment may enhance outcomes of the environmental epidemiology studies. ${ }^{8)}$ Researchers have a wide choice of portable GPS units for time-location studies. The commercial devices may differ in accuracy and precision in tracking position, ease of use, user interface, reception in various locations, data logging, processing the data, battery life, and time to acquire a signal after coming outdoors. The purpose of this study was to evaluate four low-cost commercial portable GPS devices with data logging capability for time-location assessment.

\section{Material and Method}

\section{Selection of GPS devices}

Four commercial GPS devices with data loggers were selected for the study. They were the Garmin $60 \AA$, Garmin Forerunner 201®, GeoStats GeoLogger ${ }^{\circledR}$, and Skytrx minitracker MT4100®. Criteria for the selection were the following: 1) it should be portable and convenient for subjects to carry; 2) it should have sufficient battery power to log data for at least 15 hours without replacing or recharging, so that the GPS receivers can be used to track a subject's activity outdoors in a day; 3) it should have sufficient internal memory to store at least 15 hours of timelocation data; 4) the downloaded data should be ready to use in the mapping software without any major post processing; and, 5) the cost should be 
Table 1. Description of four GPS devices

\begin{tabular}{|c|c|c|c|c|}
\hline & Garmin 60 & $\begin{array}{l}\text { Garmin Forerunner } \\
201\end{array}$ & $\begin{array}{l}\text { GeoStats } \\
\text { GeoLogger }\end{array}$ & $\begin{array}{l}\text { Skytrx minitracker } \\
\text { MT4100 }\end{array}$ \\
\hline Size $\left(\mathrm{cm}^{3}\right)$ & $6.1 \times 15.5 \times 3.3$ & $8.4 \times 4.3 \times 1.8$ & $19.1 \times 11.9 \times 8.9$ & $9.7 \times 3.8 \times 3.3$ \\
\hline $\begin{array}{l}\text { Weight }(\mathrm{g}) \text { with } \\
\text { batteries }\end{array}$ & 198.5 & 86.5 with strap & 900 & 75 \\
\hline GPS receiver & 12 channel & 12 channel & 12 channel & 12 channel \\
\hline Battery life & $\begin{array}{l}3.5 \text { days without } \\
\text { turning off the device }\end{array}$ & 15 hours & 20 hours & 4 days \\
\hline Time interval & $\begin{array}{l}\text { Programmable from } \\
1 \text { second to several } \\
\text { hours. }\end{array}$ & $\begin{array}{l}\text { from } 1 \text { second to } \\
2 \text { minutes depending } \\
\text { on the speed }\end{array}$ & $\begin{array}{l}\text { Programmable from } 5 \\
\text { seconds to } 15 \text { minutes }\end{array}$ & 1 second or more \\
\hline Internal memory & $1 \mathrm{MB}$ & Unspecified & $4 \mathrm{MB}$ & $\begin{array}{l}\text { Up to } 100 \text { hours of data } \\
\text { (about } 360,000 \text { data points) }\end{array}$ \\
\hline Data format & GDB & Tcx format & DMS & DMS \\
\hline
\end{tabular}

less than US \$500. Characteristics of the four devices are shown in Table 1.

\section{Test for accuracy and precision of stationary GPS units}

This test was conducted to determine the accuracy and precision of the devices in recording a location when placed at two known National Geodetic Survey (NGS) points are in Lexington, Kentucky, USA. The devices were placed with their antennas facing the sky at the geodetic points described in the NGS data sheets. The first location was the Campbell House hotel at the NGS coordinates 380201.61088 N-84 31 25.28919W $\left(38.03378^{\circ} \mathrm{N}-84.52369^{\circ} \mathrm{W}\right)$. The second location was the Kentucky Transportation Cabinet at the NGS coordinates of $380427.67096 \mathrm{~N}-8429$ $31.03407 \mathrm{~W}(38.07435 \mathrm{~N}-84.49195 \mathrm{~W})$. The test was repeated for 3 days at the Campbell House and for 2 days at the Kentucky Transportation Cabinet for approximately 1 hour per day at each location.

The average distance between the coordinates logged by the devices and the true coordinates of the geodetic point was calculated. In addition, the percentage of coordinates that fell within $0 \mathrm{~m}, 0-1 \mathrm{~m}$, 1-5 m, 5-10 m, and above $10 \mathrm{~m}$ were calculated for each device at each location. Finally, the distance from the geodetic point in which $95 \%$ of the coordinates were logged by each device was computed to characterize the dispersion.
3. Test for difference in logging the location when walking outdoors

This test was conducted to measure the differences across devices when they were in motion. The devices were worn as suggested by the manufacturer: the Garmin 60 was clipped to the waist belt on the right side; the GeoLogger was worn with the antenna over the right shoulder; the Garmin Forerunner was worn at the right wrist; and the Skytrx was placed in the shirt pocket.

The four devices were carried by a field technician in a pre-determined route in Lexington, Kentucky, USA. The field technician walked at a reasonably stable speed. To include different kinds of urban terrain, the approximately 2-hour route included a park, residential area, downtown area, open space, and between tall buildings. The experiment was repeated on 4 different days.

In addition, the technician repeated the walking route on each of the 4 days while holding the devices facing the sky for maximum signal reception. The four devices were fixed on a cardboard box to keep their receiving antennas facing the sky. Measures from facing the sky were assumed to be better data as this position would optimize reception, as compared with reception when worn as directed by the manufacturer.

The coordinate data from the devices was converted to degree-minute-second (DMS) format and the coordinates from each device were matched to the other devices based on the time 
logged. The two-dimensional Euclidean distance between the coordinates that were logged at the same time by any two devices was calculated. The average distances and standard error were calculated for each pair of the devices.

\section{Test for reception in a moving car}

This test was conducted to determine the differences between coordinates logged by the devices when they were placed in a moving car. The devices were placed close to the door in the left rear passenger seat of a Ford Taurus 4 door sedan. Then the vehicle was driven a $37 \mathrm{~km}$ route from 12-1PM on May 22, 2007, Lexington, Kentucky, USA. The coordinates were plotted and overlaid on the map to observe the differences in the routes logged by the devices. The coordinates that were logged at the same time by any two devices was quantitatively compared.

\section{Results and Discussion}

\section{Stationary devices}

Distributions of the distances of the coordinates logged by the Garmin 60 and the GeoLogger from the geodetic points are shown in Table 2. Only these two devices were able to save data when placed at geodetic points, as the Skytrx and

Table 2. Distribution of coordinates logged when stationary: by distance by the percentage of coordinates logged

\begin{tabular}{crrrrr}
\hline \hline $\begin{array}{c}\text { Distance in } \\
\text { meters }\end{array}$ & \multicolumn{2}{c}{ Garmin 60} & & \multicolumn{2}{c}{ GeoLogger } \\
\cline { 1 - 2 } \cline { 5 - 6 } Location & $\mathrm{CH}^{1}$ & $\mathrm{KTC}^{2}$ & & $\mathrm{CH}^{1}$ & $\mathrm{KTC}^{2}$ \\
\hline $0 \mathrm{~m}$ & $0 \%$ & $41 \%$ & & $0 \%$ & $6 \%$ \\
$0-1 \mathrm{~m}$ & $13 \%$ & $0 \%$ & & $13 \%$ & $0 \%$ \\
$1-5 \mathrm{~m}$ & $87 \%$ & $59 \%$ & & $68 \%$ & $73 \%$ \\
$5-10 \mathrm{~m}$ & $0.4 \%$ & $0 \%$ & & $17 \%$ & $19 \%$ \\
$>10 \mathrm{~m}$ & $0 \%$ & $0 \%$ & & $2 \%$ & $3 \%$ \\
95th percentile & $3.8 \mathrm{~m}$ & $2.1 \mathrm{~m}$ & & $7.7 \mathrm{~m}$ & $8.0 \mathrm{~m}$ \\
\hline
\end{tabular}

${ }^{1} \mathrm{CH}$ - Geodetic point at Campbell House; ${ }^{2} \mathrm{KTC}$ - Geodetic point at Kentucky Transportation Cabinet

*All values are the percentages of the total number of coordinates logged.

Note: Two devices did not log any points during this experiment and so are not included in the table.
Forerunner did not save coordinates when they were kept stationary. The data represent distances from the geodetic point logged in 3 days for approximately one hour each day at the Campbell House and in 2 days at the Kentucky Transportation Cabinet. Average distances from the geodetic point recorded by the Garmin 60 were $2.4 \mathrm{~m}$ (S.D. $=2.7 \mathrm{~m}$ ) at the Campbell House location and $0.9 \mathrm{~m}(\mathrm{~S} . \mathrm{D} .=0.8 \mathrm{~m})$ at the Kentucky Transportation Cabinet location. The average distances recorded by the GeoLogger were $3.5 \mathrm{~m}$ (S.D. $=3.6 \mathrm{~m}$ ) at the Campbell House location and $3.7 \mathrm{~m}$ (S.D. $=2.7 \mathrm{~m}$ ) at the Kentucky Transportation Cabinet location. The Garmin 60 and GeoLogger saved the data once every second and once every 5 seconds, respectively.

The Garmin 60 had more accurate readings than the GeoLogger. Of all readings by the Garmin 60 at the Campbell House, 13\% were logged less than $1 \mathrm{~m}$ from the geodetic point, and of those at the Kentucky Transportation Cabinet location, $41 \%$ were within $1 \mathrm{~m}$. For the GeoLogger, these coordinates were $13 \%$ at the Campbell House location and $6 \%$ at the Kentucky Transportation Cabinet location. More than $99 \%$ of readings from the Garmin 60 were logged at less than $5 \mathrm{~m}$ at both geodetic points, whereas $81 \%$ and $79 \%$ of the GeoLogger readings were logged at less than $5 \mathrm{~m}$ at the Campbell House location and the Kentucky Transportation Cabinet location, respectively. Ninetyfive percent of the Garmin 60 coordinates were within $3.8 \mathrm{~m}$ and $2.1 \mathrm{~m}$ from the geodetic points at the Campbell House and the Kentucky Transportation Cabinet, respectively. Ninety-five percent of the GeoLogger coordinates were within $7.7 \mathrm{~m}$ and $8.0 \mathrm{~m}$ from the geodetic points at the Campbell House and the Kentucky Transportation Cabinet, respectively.

\section{Devices used while walking}

Table 3 shows the distances between each pair of devices measured during a pre-determined walking route. The coordinates were matched based on the time recorded in the downloaded data of each device without any correction. When the devices were facing the sky, the Forerunner, the GeoLogger, and the Skytrx readings were logged at mean distances of $4.7 \mathrm{~m}, 4.9 \mathrm{~m}$, and $18.2 \mathrm{~m}$ 
Table 3. Comparison of the distance between the coordinates logged by each pair of devices when worn while walking. The devices were either kept in a box facing the sky or worn as directed by the manufacturer

\begin{tabular}{|c|c|c|c|c|c|}
\hline \multirow[b]{2}{*}{ Device 1} & \multirow[b]{2}{*}{ Device 2} & \multicolumn{2}{|c|}{ Facing sky } & \multicolumn{2}{|c|}{ Wearing } \\
\hline & & Mean distance $(\mathrm{m})$ & Standard deviation & Mean distance $(\mathrm{m})$ & Standard deviation \\
\hline Garmin 60 & Forerunner & 4.7 & 12.2 & 8.2 & 6.9 \\
\hline Garmin 60 & GeoLogger & 4.9 & 11.1 & 6.8 & 7.7 \\
\hline Garmin 60 & Skytrx & 18.3 & 7.1 & 14.1 & 6.0 \\
\hline Forerunner & GeoLogger & 7.0 & 17.9 & 8.1 & 5.2 \\
\hline Forerunner & Skytrx & 17.8 & 5.2 & 16.7 & 6.7 \\
\hline GeoLogger & Skytrx & 18.9 & 12.7 & 15.4 & 9.0 \\
\hline Garmin 60 & Skytrx Adjusted* & 6.2 & 8.3 & 9.0 & 7.3 \\
\hline Forerunner & Skytrx Adjusted* & 6.6 & 4.7 & 8.0 & 6.6 \\
\hline GeoLogger & Skytrx Adjusted* & 7.4 & 11.9 & 10.5 & 7.4 \\
\hline
\end{tabular}

*: Coordinates of Skytrx were adjusted by 10 seconds.

from the coordinates logged by the Garmin 60, respectively. The Skytrx readings differed by mean distances of $17.8 \mathrm{~m}$ and $18.9 \mathrm{~m}$ from the coordinates logged by the Forerunner and the GeoLogger, respectively. The Forerunner and the GeoLogger logged coordinates with a mean distance of $7 \mathrm{~m}$ from each other.

Although the coordinates logged by the Skytrx facing the sky were about $19 \mathrm{~m}$ from the other devices, mapping of the coordinates from all the devices showed a similar route. Detailed mapping revealed that the coordinates by Skytrx were the same as the other devices, but the internal time of Skytrx may have been ahead of those of the other devices. When the Skytrx coordinates were adjusted by 10 seconds, the distance between the Skytrx and Garmin 60 was reduced to $6.2 \mathrm{~m}$.

The mean distances between the coordinates logged by the Garmin 60, the Forerunner, and the GeoLogger increased when the devices were worn as the manufacturer suggested compared to when the devices were carried with antennas facing the sky. When the devices were worn as suggested, the Forerunner and the GeoLogger readings were logged at mean distances of $8.2 \mathrm{~m}$ and $6.8 \mathrm{~m}$ from the coordinates logged by the Garmin 60, respectively. The Skytrx readings were closer to other devices when it was worn by subject. The Skytrx readings differed by mean distances of $14.1 \mathrm{~m}, 16.7 \mathrm{~m}$ and $15.4 \mathrm{~m}$ from the coordinates logged by the Garmin 60 , the Forerunner and the GeoLogger, respectively.
The distances from the Garmin 60, Forerunner and Geologger were reduced to $9.0,8.0$ and $10.5 \mathrm{~m}$ when the internal time of the Skytrx was adjusted by 10 seconds. When the internal time of the Skytrx was adjusted, mean distances of coordinates between the Skytrx and the other devices slightly increased when the devices were worn as suggested.

The results from this study confirm that in most instances, GPS devices provide reasonably accurate and sufficient data for the purposes of exposure assessment. For instance, two of the devices were accurate within $4 \mathrm{~m}$ when stationary. Three devices were accurate within $5 \mathrm{~m}$ when worn as directed by the manufacturer. Such accuracy up to $10 \mathrm{~m}$ can provide reasonably correct location information. For example, distances between houses or buildings are more than $10 \mathrm{~m}$. Tests for reception revealed some loss of data. But the Skytrx, even at reduced rates, logged about 31 coordinates per minute, which would be good enough for typical exposure analysis studies. The Forerunner logged at a rate of about 2 coordinates per minute when it was worn.

As in other studies, ${ }^{5,7)}$ the GPS devices experienced instances of failure to collect data, and collected data that was inaccurate or insufficient. Notably, the Garmin Forerunner and the Skytrx minitracker did not record coordinates when they were stationary. The distances between the coordinates logged by combinations involving the Skytrx with internal time adjusted by 10 seconds were 
significantly higher when the devices were worn by a person $(p<0.0001)$. The reason for this difference may be the variable interference of the subject's body in the signal reception of the devices. When the internal time was adjusted by 10 seconds, distances between the Skytrx and Garmin 60 were reduced significantly. When tested in a car, distances between the Skytrx and Garmin 60 were reduced significantly with time adjustment. However, such difference may not be significant for exposure analysis study.

\section{Devices used in a car}

When the devices were placed in a car, the Forerunner, the GeoLogger, and the Skytrx readings were logged at mean distances of $7.1 \mathrm{~m}, 11.1 \mathrm{~m}$ and $140 \mathrm{~m}$ from the coordinates logged by the Garmin 60, respectively. When the Skytrx readings were adjusted by 10 seconds, the distance from the Garmin 60 was $42 \mathrm{~m}$. When the maps by the four devices were overlaid, they showed identical routes.

The number of coordinates logged per hour was calculated for each device. The reception rates are shown in Table 4. The reception rates of all the devices decreased when they were worn as suggested compared to the rates when they were carried in a box or placed in the car. The reception rates for the Garmin 60, the GeoLogger, and the Skytrx increased when they were placed in the car compared to when they were carried with the antennas facing the sky. However, the logging rate of the Forerunner was reduced when it was placed in the car.

\section{Field usability}

User friendliness is an important characteristic for these devices. The GeoLogger is a 3 piece

Table 4. Coordinate logging rates per minute for each device when walking and driving

\begin{tabular}{lccc}
\hline \hline \multicolumn{1}{c}{ Device } & $\begin{array}{c}\text { Walking } \\
\text { (Facing sky) }\end{array}$ & $\begin{array}{c}\text { Walking } \\
\text { (Wearing) }\end{array}$ & Driving \\
\hline Forerunner & $4.1(100 \%)$ & $2.4(60 \%)$ & $3.7(90 \%)$ \\
Garmin 60 & $56.7(100 \%)$ & $53.3(94 \%)$ & $57.7(101 \%)$ \\
GeoLogger & $10.4(100 \%)$ & $10.1(97 \%)$ & $11.6(111 \%)$ \\
Skytrx & $48.7(100 \%)$ & $30.8(63 \%)$ & $48.8(100 \%)$ \\
\hline
\end{tabular}

device. Similar to the GPS unit used in other studies, ${ }^{5,7)}$ this type of device may not be comfortable for users. Furthermore, subjects may not be willing to hold the Geologger's antenna towards the sky to get a good signal reception. This may be a potential problem with subject cooperation. Although the GeoLogger provided reasonable accuracy, it may not be suitable for exposure analysis studies.

It may be critical to have minimal intervention from study subjects. The Forerunner may also pose challenges for study subjects because it requires extensive user input during monitoring. The device shuts down automatically when the subject comes indoors. Since it should be restarted after coming outdoors, this would lead to some data loss if the subject forgets to turn it on again. Restarting takes about 2 minutes. It will be too much demand from the subjects to restart the device every time they go outdoors. In addition, the rate of logging the coordinates is two per minute and unchangeable. This may be a limitation when the subjects are moving at higher speeds.

\section{Summary}

GPS devices automatically record study subjects' time and location, relieving them of the responsibility of accurately recalling and recording the information a diary or survey. With rapid advances in GPS technology, the new devices are half the weight and the cost compared to those available just five years ago. ${ }^{5)}$ Exposure assessment scientists have a choice of many new lightweight and affordable devices for their research. Our objective assessment of four of these devices provides valuable information for researchers planning field studies. We found that the Garmin 60 performed well in all our tests. The Skytrx also provided accurate information when the internal time of the device is adjusted. Even without the adjustment, the Skytrx preformed well from the perspective of exposure analysis.

Results from the experiments described in this paper suggest that miniature, portable, low-cost GPS receivers can be used to track study subjects' time and activity, and therefore can be used to assess transient exposure to pollutants both spatially and temporally. We conclude that the Garmin 60 
and Skytrx devices can be used for time-location measurement in exposure analysis.

\section{Acknowledgement}

Authors do not have any conflict of financial interest. The project was supported by EPA STAR grant (RD-83154001).

\section{References}

1. Freeman, N. C. G., Tejada, S. S. D. : Methods for collecting time/activity pattern information related to exposure to combustion products. Chemosphere, 49, 979-992, 2002.

2. Robinson, J., Godbey, G. : Time for Life, Penn State Press, 1997.

3. Kaplan, E. : Understanding GPS: Principles \& Applications, Artech House, 1996.

4. US EPA, GIS Technical Memorandum 3: Global positioning systems technology and its application in environmental programs. Washington, DC., 1992.

5. Elgethun, K., Fenske, R., Yost, M., Palcisko, G. : Time-location analysis for exposure assessment studies of children using a novel global positioning system instrument. Environmental Health Perspectives, 111, 115-122, 2003.

6. Elgethun, K., Yost, M., Fitzpatrick, C., Nyerges, T., Fenske, R. : Comparison of global positioning system (GPS) tracking and parent-report diaries to characterize children's time-location patterns. Journal of Exposure Science and Environmental Epidemiology, 17, 196-206, 2007.

7. Phillips, M., Hall, T., Esmen, N., Lynch, R., Johnson, D. : Use of global positioning system technology to track subject's location during environmental exposure sampling. Journal of Exposure Analysis and Environmental Epidemiology, 11, 207215,2001

8. Nuckols, J. R., Ward, M. H., Jarup, L. : Using geographic information systems for exposure assessment in environmental epidemiology studies. Environmental Health Perspectives, 112, 1007-1015, 2004. 